\title{
Preparatory strategies in overlapping-task performance
}

\author{
RITSKE DE JONG \\ University of Groningen, Groningen, The Netherlands \\ and \\ JOHN B. SWEET \\ Ohio State University, Columbus, Ohio
}

\begin{abstract}
In four experiments, we tested whether perceptual identification is impeded by temporal overlap with another task. The results demonstrate that temporal overlap with an auditory task consistently impaired the identification of the highest digit in a briefly presented and masked array of digits. The impairment was especially pronounced when we emphasized speed on the auditory task, thus counteracting the normal tendency to focus advance preparation on the more challenging visual task. The data also indicate that previous findings of minimal effects of temporal overlap may have been due to the use of a restricted range of stimulus onset asynchronies. The present results demonstrate the important role of preparatory strategies in overlapping-task performance, and show that perceptual identification is impeded by the overlapping performance of another task.
\end{abstract}

In the psychological refractory period (PRP) paradigm, two stimuli are presented at a fixed or variable stimulus onset asynchrony (SOA), with each stimulus requiring a separate response. Typically, the response to the second stimulus is prolonged, compared with when it is presented in isolation, and is more so the shorter the SOA. This socalled PRP effect appears to reflect a fundamental limitation in subjects' ability to perform two discrete tasks simultaneously.

The PRP effect is commonly attributed to the inability of a central processing mechanism, or channel, to engage in more than one task or operation at a time. Because of this limitation, component processes in the second task that require the central channel must wait until the channel has been cleared by the first task. The central channel thus constitutes a structural bottleneck that greatly limits the extent to which two tasks can be performed in parallel.

Much evidence indicates that response selection is subject to postponement in the PRP paradigm, suggesting that one function of the central channel is to select a response based on the output of perceptual processes and the applicable input-output mapping (McCann \& Johnston, 1992; Pashler, 1984; Pashler \& Johnston, 1989; Welford, 1980). In contrast, at least some types of perceptual processes seem capable of proceeding in parallel with another task (Pashler, 1984; Pashler \& Johnston, 1989). Perhaps the most impressive evidence that perceptual processing is not subject to postponement has recently been reported

We thank Robert McCann, Harold Pashler, and Lester Krueger for helpful suggestions. Correspondence concerning this article should be addressed to R. De Jong, Department of Experimental and Occupational Psychology, University of Groningen, Grote Kruisstraat 2/1, 9712 TS Groningen, The Netherlands.

-Accepted by previous editor, Charles $W$. Eriksen by Pashler (1989). In an interesting variant of the PRP paradigm, Pashler used a perceptually demanding second task in which the complex visual stimulus was only briefly presented and then masked. The response in the visual task was unspeeded and its accuracy was measured as a function of SOA. In one task, the stimulus consisted of a briefly presented and subsequently masked $2 \times 4$ array of digits, and the task was to identify the highest digit in the array.

Accuracy in identifying the highest digit was only minimally affected by overlap with an auditory reaction time (RT) task with two highly discriminable tones, but was strongly affected by overlap with a visual task that also required complex perceptual processing. Evidently, the perceptual processing required in the highest-digit task, including the identification of the array elements, could take place in parallel with another task, but only if the latter task imposed minimal perceptual requirements in the same modality. Pashler (1989) concluded that perceptual processing is not subject to postponement, but can be interfered with by other ongoing perceptual processes in the same modality; that is, it has resource-like rather than bottleneck-like properties.

However, other evidence suggests that identification of visual stimuli may have to be postponed while the subject is engaged in an auditory RT task. De Jong (1993) used the diagnostic of underadditive interactions between the effects of SOA and manipulations of perceptual factors in the visual, second RT task in order to determine whether perceptual processing in that task could proceed while the central channel was occupied by the auditory, first task (Pashler, 1984; Schweickert, 1978). Early perceptual processes, for example, those affected by stimulus intensity, were not subject to postponement. In contrast, stimulus identification and categorization were found 
to be postponed, suggesting that these perceptual processes required the central channel. On the basis of these results, De Jong (1993) concluded that the central channel plays a crucial role in the construction of categorical or propositional codes, whether of perceptual inputs, results of intermediate cognitive operations, or responses. He also suggested that its function might be to prepare the relevant processing mechanisms for the computation of such codes, rather than to actively participate in such computations.

These latter results appear to conflict with those reported by Pashler (1989). Performing the highest-digit task well above chance requires identification of at least several of the digits in the display. Moreover, because the display was only briefly presented and then masked, such identification presumably overlapped at short SOAs with processing in the auditory task. The results of De Jong (1993) would therefore lead one to expect a marked decrement in the ability to identify the highest digit at short SOAs. Instead, Pashler (1989) found that such decrements were quite minimal. In the experiments reported here, we set out to determine the cause of this discrepancy. Resolution of this issue is important because the conflicting findings preclude at present any firm conclusions regarding the time course and processing requirements of perceptual identification in overlapping-task performance.

\section{Preparatory Strategies in \\ Overlapping-Task Performance}

The different results regarding perceptual processing in the visual, second task reported by Pashler (1989) and De Jong (1993) were paralleled by a marked difference in auditory-task performance. Using tones of 300 and $900 \mathrm{~Hz}$, Pashler obtained mean RTs well over $450 \mathrm{msec}$; De Jong used less discriminable tones of 1000 and $1035 \mathrm{~Hz}$, but obtained mean RTs well under $400 \mathrm{msec}$. These results suggest that different strategies were adopted by the subjects in the two studies.

We propose that such strategies can be characterized by how a limited capacity for advance preparation of the processing system for specific operations is divided in a graded manner among multiple tasks. This proposal is closely related to the idea of graded capacity sharing in dual-task performance (Kahneman, 1973; McLeod, 1977). In order to explain the conflicting evidence regarding the question of parallel perceptual identification, we further assume that both perceptual identification and response selection can operate only to the extent that these processes have been prepared in advance (Gottsdanker, 1979, 1980; Logan, 1978). Focusing advance preparation on the auditory task should then prevent the identification of the visual stimulus from proceeding until the first response has been selected and preparation can be switched to the visual task. Such a strategy would account for the results of De Jong (1993). Alternatively, dividing advance preparation among the two tasks should hurt performance of the auditory task, but allow identification of the visual stimulus to proceed in parallel with the auditory task. The results reported by Pashler (1989) are consistent with the latter strategy. These different strategies would seem to make good functional sense because De Jong used visual stimuli that remained on until a response was made, whereas Pashler used brief and masked stimuli. The first two experiments reported here tested this explanation of the discrepant findings of Pashler (1989) and De Jong (1993) by explicitly manipulating relative task emphasis for the auditory and highest-digit task combination studied by Pashler.

Before turning to these experiments, it is useful to elaborate this proposal in more detail. There is compelling evidence that two distinct factors contribute to interference in overlapping-task performance: (1) preparatory limitations (De Jong, in press; Gottsdanker, 1979, 1980) and (2) structural processing limitations, or bottlenecks (Pashler, 1989, in press). However, the relative contributions of these two factors have yet to be adequately assessed. We propose that these contributions depend critically on the preparatory strategies that subjects employ. When both tasks are prepared in advance, structural limitations of specific processing mechanisms will constitute the primary limiting factors in overlapping-task performance. It is under such conditions that distinct and qualitatively different types of processing limitations for perceptual mechanisms and for response-selection mechanisms become evident, the former being resource-like and the latter bottleneck-like (Pashler, 1989, in press). When advance preparation is focused on the first task, perceptual identification and response selection in the second task will not be postponed due to structural processing limitations, but due to the fact that the processing system is temporarily unprepared for these operations (De Jong, in press; Gottsdanker, 1979, 1980; McCann \& Johnston, 1992). In this view, the conclusions that perceptual and responseselection interference have a common origin (De Jong, 1993) or that perceptual processing in the second task is unaffected by temporal overlap if the perceptual requirements of the first task are minimal (Pashler, 1989) are only partially correct. To properly assess the actual causes of observed interference between overlapping tasks, preparatory strategies must be taken into account.

\section{EXPERIMENT 1}

In this experiment, we employed the same choice RT auditory task and highest-digit task combination that was used by Pashler (1989). Relative task emphasis was manipulated by explicit instructions and by bonus systems that differentially rewarded performance on either task. According to the present hypothesis, this manipulation should reveal a distinct reciprocity between the two tasks. Emphasis on the auditory task should induce subjects to focus advance preparation on this task, resulting in relatively fast responses to the tones and, especially at short SOAs, in relatively low accuracy in the digit task. Emphasis on the digit task, on the other hand, should induce subjects to focus advance preparation relatively more on the digit task, resulting in slower responses to the tones but, especially at short SOAs, in higher accuracy in the digit task. 


\section{Method}

Subjects. Seven undergraduates ( 3 male, 4 female) were paid $\$ 2.50 / \mathrm{h}$ plus bonuses. Data were excluded for 1 other subject, who made an unacceptable number of errors $(21 \%)$ in the auditory task when that task was de-emphasized.

Apparatus and Stimuli. Two tones (1000 and $1035 \mathrm{~Hz}, 65-\mathrm{dB}$ amplitude), generated by a Schlumberger sine-square audio generator (Model SG-18A), served as stimuli in the auditory task. An array of eight digits in two rows of four, presented on a DEC VT-11 CRT display, served as the stimulus in the second task. The array was centrally located around a fixation point. The highest digit was selected randomly from the range of 6-9, and the seven distractors were selected randomly from 1 to the highest digit. Note that the highest digit could be repeated in the array. The digits were randomly assigned to the positions in the array. The eight elements of the mask array were constructed separately by superimposing eight digits that were randomly selected, with replacement, from the range of $1-5$. New mask elements were constructed on every trial. The digits subtended about $0.6^{\circ}$ of visual angle, and the arrays measured about $3.0^{\circ} \times 1.5^{\circ}$ of visual angle from a viewing distance of $1 \mathrm{~m}$.

Design. The subjects participated in three sessions, with the first session used for instruction and training. There were 16 blocks in each session of $\mathbf{4 8}$ trials each. Relative task emphasis was reversed after every 4 blocks.

Procedure. Presentation of the central fixation point signaled the beginning of a trial, and was followed after $500 \mathrm{msec}$ by the presentation of one of the tones for $100 \mathrm{msec}$. Tone onset was followed by the onset of the digit display after one of two equiprobable SOAs (10 or $900 \mathrm{msec}$ ). The mask display was presented for $250 \mathrm{msec}$ immediately after the digit display duration had expired.

Display duration was varied on a trial-by-trial basis; it was shortened by $7 \mathrm{msec}$ after a correct digit response and lengthened by $13 \mathrm{msec}$ after an incorrect response. This resulted in an average accuracy of about $65 \%$ in the digit task, irrespective of relative task emphasis. Because the SOAs were randomly distributed across trials, this procedure did not confound display duration and SOA. Separate durations were maintained for the two task-emphasis conditions. The initial durations were set at $250 \mathrm{msec}$ at the beginning of the first session; the final values at the end of each session became the initial values for the next session.

Half a second after the mask had expired, the subject was prompted to select the highest digit by pushing one of four buttons, corresponding to $6,7,8$, or 9 . The subjects were allowed up to $10 \mathrm{sec}$ to make this response, and the next trial started $1 \mathrm{sec}$ after it had been recorded.

A bonus system was used to manipulate relative task emphasis. Under auditory-task emphasis, the subjects earned 1.54 if the tone response was faster than a criterion, and lost the same amount if it was slower. The criterion was periodically updated so that each subject would be able to beat it on about $65 \%$ of the trials. The subjects earned $0.7 \uparrow$ for a correct digit response and lost 0.74 for an error. Under digit-task emphasis, they won or lost $0.7 \$$ in the auditory task and 1.54 in the digit task. An error in the auditory task always cost $3 \leftarrow$. The bonus systems and their rationale were carefully explained to the subjects. They were told that the speed criterion for the auditory task was set to different values, depending on relative task emphasis, but they were not informed that the display duration and speed criterion were continually updated on the basis of their performance, and none reported having been aware of this. The subjects earned an average bonus of about $\$ 4 / \mathrm{h}$.

\section{Results}

Average correct auditory RTs, percent errors in the two tasks, and display durations are presented in Table 1 , for each combination of task emphasis and SOA. Display durations were averaged across the two experimental sessions.

Auditory (first) task. Mean RTs were $41 \mathrm{msec}$ faster when the auditory task was emphasized $[F(1,6)=23.27$, $p<.01]$. Errors were also more frequent in this condition $[F(1,6)=11.31, p<.02]$. Thus, the faster responses in this condition seem to have been achieved, at least in part, by trading accuracy for speed.

Digit (second) task. Display durations were somewhat shorter when the digit task was emphasized $[F(1,6)=$ $15.22, p<.01]$, indicating that the same level of performance was achieved with a shorter display duration in that condition. More errors were made at the short SOA $[F(1,6)=18.85, p<.01]$. Emphasis did not affect error rate $(F<1)$; this simply reflects the fact that, as intended, the trial-to-trial tracking of display duration resulted in the same overall error rate for the two emphasis conditions. Most importantly, the difference in error rate between the two SOAs was larger when the tone task was emphasized, as indicated by a significant interaction of task emphasis and SOA $[F(1,6)=14.17, p<.01]$. A separate analysis revealed that the $9.5 \%$ SOA effect obtained under digit-task emphasis was significant $[F(1,6)=$ $10.86, p<.02$ ].

In order to assess possible trial-to-trial dependencies between the tasks, we computed average auditory RTs con-

Table 1

Mean Reaction Time (RT), Display Duration, and Error Rates in the Auditory and Visual Tasks as a Function of Task Emphasis and SOA in Experiment 1

\begin{tabular}{|c|c|c|c|c|}
\hline & \multicolumn{4}{|c|}{ Task Emphasis } \\
\hline & \multicolumn{2}{|c|}{ Auditory } & \multicolumn{2}{|c|}{ Visual } \\
\hline & 10-msec SOA & $900-\mathrm{msec} \mathrm{SOA}$ & 10-msec SOA & 900-msec SOA \\
\hline \multicolumn{5}{|c|}{ Auditory Task } \\
\hline RT & 345 & 349 & 391 & 386 \\
\hline Percent errors & 7.1 & 6.7 & 5.4 & 4.1 \\
\hline \multicolumn{5}{|c|}{ Visual Task } \\
\hline Display duration & 148 & 148 & 139 & 139 \\
\hline Percent errors & 41.6 & 27.0 & 37.8 & 28.3 \\
\hline
\end{tabular}

Note-RTs and display durations are in milliseconds. 
Table 2

Mean Tone Reaction Time as a Function of Task Emphasis, SOA, and the Accuracy of the Digit Response in Experiment 1

\begin{tabular}{cccccc}
\hline & \multicolumn{4}{c}{ Task Emphasis } \\
\cline { 2 - 5 } \cline { 5 - 6 } Digit Response & \multicolumn{3}{c}{ Auditory } & \multicolumn{2}{c}{ Visual } \\
\cline { 2 - 6 } \cline { 5 - 6 } 10-msec SOA & 900 -msec SOA & & 10-msec SOA & 900 -msec SOA \\
\hline Correct & 340 & 349 & 388 & 388 \\
Incorrect & 352 & 350 & 395 & 382 \\
\hline
\end{tabular}

Note-RTs are in milliseconds.

ditionalized on whether the digit response was correct or incorrect. The results of this analysis are shown in Table 2 . Neither the main effect of digit response correctness nor any of the interactions involving this factor approached significance.

\section{Discussion}

The results exhibit the predicted reciprocity between the two overlapping tasks. Emphasis on the auditory task resulted in (1) faster though somewhat less accurate responses in that task, (2) a decrease in overall performance in the digit task; that is, longer display durations were required to achieve the same overall accuracy, and (3) a larger detrimental effect of temporal overlap on accuracy in the digit task. Thus, when the subjects were induced to focus advance preparation on the auditory task, performance of the digit task deteriorated markedly at short SOAs, that is, when the tasks overlapped extensively. These results indicate that the identification of the digits was impeded when the processing system was temporarily not adequately prepared for the digit task. This is consistent with the notion that perceptual identification requires explicit preparation (De Jong, 1993).

Performance in the digit task exceeded chance levels, even under the least favorable conditions. This does not contradict the notion that perceptual identification cannot proceed unless prepared in advance, because, as suggested by the subjects' reports, the emphasis on the auditory task may not have succeeded in diverting advance preparation entirely away from the more challenging digit task. Furthermore, it remains unclear to what extent masking of long-duration displays, such as the ones used here, can obliterate the results of early perceptual processing (Eriksen, 1980). The important finding, we suggest, is that the detrimental effect of temporal overlap on digit-task performance was significantly influenced by the degree to which the digit task was prepared in advance.

We found no evidence for trial-to-trial dependencies between the two tasks. This contrasts with the clear tradeoffs found when instructions emphasized one task over the other. There are several possible reasons for this dissociation between what may be called microdependencies and macrotradeoffs. Systematic and trial-to-trial fluctuations in relative task emphasis are assumed to produce negative macrotradeoffs and negative microdependencies, respectively. However, positive microdependencies would be predicted if there were trial-to-trial fluctuations in overall preparedness and performance, or if swift completion of the auditory task at short SOAs provided time to switch preparation to the digit task before the display was masked. The net effect of these three factors (one negative, two positive) on the form of the microdependencies is difficult to predict. Pashler (1989) found positive microdependencies, as did we in two of the experiments reported here. In two other experiments, including the present one, we found a nonsignificant trend toward positive microdependencies. These somewhat variable results should not be surprising, given the various competing factors that may affect microdependencies. ${ }^{1}$ Perhaps most importantly, the present results indicate that the effects of preparatory strategies on overlapping-task performance are best assessed by manipulation through explicit instructions, that is, by examining macrotradeoffs.

The difference in error rate between the two SOAs when the digit task was emphasized was a sizable $9.5 \%$, which far exceeded the $2.5 \%$ reported by Pashler (1989). However, even when the digit task was emphasized, tone responses were still about $100 \mathrm{msec}$ faster than those reported by Pashler (1989), despite the fact that we used less discriminable tones. Given the observed reciprocity between tasks, de-emphasizing the auditory task even further might have resulted in an even smaller effect of temporal overlap on the accuracy in the digit task. However, there are several procedural differences between the two experiments that also may account for the larger SOA effect in the present experiment.

In the following experiments, we examined the influence of some of these differences: the amount of practice received by subjects, the type of mask used to delimit visual processing time, and the set and range of the SOAs.

\section{EXPERIMENT 2}

The failure to replicate Pashler's (1989) results in Experiment 1 , even when the digit task was emphasized, may have been due to our use of a within-subject design with highly practiced subjects. In Experiment 2, therefore, we used unpracticed subjects and manipulated relative task emphasis as a between-subject factor. Half of the subjects received instructions that did not specifically emphasize either task. These subjects were expected to focus advance preparation primarily on the more challenging digit task and to perform rather poorly in the auditory task. The auditory task was emphasized for the other subjects by means of a bonus system, as described below. In addition, the tones were made less discriminable for the latter subjects, in a further attempt to induce them to focus preparation on the auditory task. 


\section{Method}

Subjects. Twenty-eight Ohio State University undergraduates participated for course credit.

Apparatus and Stimuli. The visual stimuli were presented on Seiko CM-1440 color monitors, controlled by IBM-compatible microcomputers (equipped with VGA graphics, providing a display resolution of $640 \times 480$ pixels). The digit and mask displays were constructed as in Experiment 1, and presented in white on a black background. Digits and masks were about $0.5 \mathrm{~cm}$ wide and $0.8 \mathrm{~cm}$ high, and the total array measured $4.0 \times 2.5 \mathrm{~cm}$, subtending $3.8^{\circ} \times 2.4^{\circ}$ of visual angle, based on a typical viewing distance of $60 \mathrm{~cm}$. The tones for the first, auditory task were presented through the speaker of the microcomputer, at 575 or $625 \mathrm{~Hz}$ when the tone task was emphasized, and at 300 and $900 \mathrm{~Hz}$ when neither of the two tasks was specifically emphasized.

Design. The subjects served in a single session of about $1 \mathrm{~h}$. They performed 16 blocks of 32 trials each. The first 2 blocks were considered training and were excluded from analysis. Half of the subjects performed under auditory-task emphasis and the other half were not explicitly induced to emphasize either task.

Procedure. Onset of a 500-msec duration plus sign as a central fixation point signaled the beginning of a trial; $500 \mathrm{msec}$ after its offset, one of the two tones was presented for $100 \mathrm{msec}$. After one of two equiprobable SOAs, 50 or $1,000 \mathrm{msec}$, the digit display appeared at the center of the display, and immediately after its duration had expired, it was replaced with masks for $250 \mathrm{msec}$. The subjects responded to the tone by pressing either the $\mathrm{Z}$ or $\mathrm{X}$ key on the keyboard, corresponding to a low or a high tone, respectively, using the middle finger or index of the left hand. They indicated the highest digit by pressing the $B, N, M$, or comma key, corresponding to $6,7,8$, or 9 , respectively. The digit response was accepted only after the appearance of a prompt, which appeared $500 \mathrm{msec}$ after offset of the mask display.

Two independent tracking procedures produced two different exposure durations for the digit display. The short duration was increased by $12 \mathrm{msec}$ after an incorrect digit response and decreased by $8 \mathrm{msec}$ after a correct response. The long duration was increased or decreased by 16 and $4 \mathrm{msec}$, respectively. The duration was thus controlled to result, for each individual subject, in an average accuracy of $60 \%$ in the short-duration digit task and $80 \%$ in the longduration digit task. The two durations were equiprobable and randomly distributed across trials. The short and long durations were initially set at 250 and $400 \mathrm{msec}$, respectively. Because tone fre- quency, the SOA, and the highest digit were also randomly and independently varied across trials, short versus long display duration was never confounded with either of these variables.

Written instructions stated that the tone response should be given as rapidly and accurately as possible, while accuracy only was stressed for the digit task. For half of the subjects, the auditory task was further emphasized by the following bonus system. The subjects in this condition earned 14 for a fast tone response and lost $1 \$$ for a slow tone response, according to a criterion time that was periodically updated so that each subject would beat the criterion on about $70 \%$ of the trials. The subjects lost $2 థ$ for each error in the auditory task. They earned $0.5 \uparrow$ for a correct digit response and lost 0.54 for an error. Feedback about the speed ("FAST" or "SLOW") and accuracy of the tone response and the digit response, as well as the bonus earned or lost for each response, was displayed for $1 \mathrm{sec}$ at the end of each trial, followed by a 250 -msec offset. When the tone response exceeded $500 \mathrm{msec}$, an additional warning message ("TONE RESPONSE TOO SLOW') was displayed for $750 \mathrm{msec}$, followed by a $250-\mathrm{msec}$ offset. At the end of a block, the subjects received feedback about the mean correct RT for the auditory task, the number of errors on each task, and the total bonus earned in the block. They then rested until ready to resume. The subjects in this condition earned an average bonus of about $\$ 2$.

No bonus system was used for the subjects in the no-emphasis condition. If an error was made on either task in this condition, a warning message ("ERROR') was displayed for $750 \mathrm{msec}$, followed by a 250 -msec offset. Feedback was provided at the end of a block, which consisted of mean correct RT for the auditory task and the number of errors on each task.

\section{Results}

Mean RTs and percent errors in the auditory task and mean display durations and errors in the digit task are presented in Table 3, for each combination of task emphasis, short versus long display duration, and SOA.

Auditory (first) task. Mean RTs were $99 \mathrm{msec}$ faster when the auditory task was emphasized $[F(1,26)=6.97$, $p<.02]$. No other effects on RT approached significance. More errors were made when the auditory task was emphasized $[F(1,26)=7.31, p<.02]$. Also, more er-

Table 3

Mean Reaction Time (RT), Display Duration, and Error Rates

in the Auditory and Visual Tasks as a Function of Task Emphasis, Short Versus Long Display Duration, and SOA in Experiment 2

\begin{tabular}{|c|c|c|c|c|}
\hline & \multicolumn{4}{|c|}{ Task Emphasis } \\
\hline & \multicolumn{2}{|c|}{ Auditory } & \multicolumn{2}{|c|}{ Auditory and Visual } \\
\hline & 50-msec SOA & 1,000 -msec SOA & 50-msec SOA & 1,000 -msec SOA \\
\hline \multicolumn{5}{|c|}{ Short Display Duration: Auditory } \\
\hline RT & 413 & 413 & 521 & 511 \\
\hline Percent errors & 8.5 & 8.5 & 5.6 & 3.3 \\
\hline \multicolumn{5}{|c|}{ Short Display Duration: Visual } \\
\hline Display duration & 261 & 261 & 214 & 214 \\
\hline Percent errors & 46.5 & 27.6 & 42.9 & 31.1 \\
\hline \multicolumn{5}{|c|}{ Long Display Duration: Auditory } \\
\hline RT & 409 & 415 & 504 & 507 \\
\hline Percent errors & 8.6 & 7.6 & 6.1 & 3.5 \\
\hline \multicolumn{5}{|c|}{ Long Display Duration: Visual } \\
\hline Display duration & 439 & 441 & 377 & 375 \\
\hline Percent errors & 27.3 & 17.2 & 23.2 & 18.3 \\
\hline
\end{tabular}

Note-RTs and display durations are in milliseconds. 
rors were made at the short SOA $[F(1,26)=10.64, p<$ $.01]$. The latter effect appeared to be present mainly in the no-emphasis condition, as indicated by a significant interaction of task emphasis and SOA $[F(1,26)=4.34$, $p<.05]$.

Digit (second) task. Mean display durations were $55 \mathrm{msec}$ shorter in the no-emphasis condition, but this effect did not approach significance $[F(1,26)=1.42, p<$ $.24]$. The long display durations resulted, of course, in much lower error rates in the digit task $[F(1,26)=$ $397.36, p<.001]$. More importantly, errors in the digit task occurred more frequently at the short SOA $[F(1,26)=$ $68.32, p<.001]$, especially when the auditory task was emphasized, as indicated by a significant interaction of task emphasis and SOA $[F(1,26)=4.95, p<.04]$. The SOA effect on error rate was also stronger for short display durations, as indicated by a significant interaction of short versus long display duration and SOA $[F(1,26)=$ $17.97, p<.001]$. No other effects approached significance. A separate analysis of the no-emphasis condition revealed a main effect of SOA $[F(1,13)=28.60, p<$ $.001]$ and a significant interaction of short versus long display duration and SOA $[F(1,13)=7.54, p<.02]$.

In Table 4, mean tone RTs are conditionalized on the accuracy of the digit response. Tone responses were faster when the digit response was correct, a positive microdependency $[F(1,26)=23.70, p<.001]$. This effect was significantly stronger at the short SOA, as indicated by a significant interaction of SOA and digit accuracy $[F(1,26)=7.64, p<.02]$, and was also stronger for the long display duration $[F(1,26)=4.33, p<.05]$.

\section{Discussion}

In this experiment, relative task emphasis was manipulated as a between-subject factor and the subjects received very little practice. Even so, the results were similar to those obtained in Experiment 1, which employed a within-subject design with highly practiced subjects. Tone responses were faster when instructions emphasized the auditory task, despite the fact that the two tones were less discriminable in this condition. As in Experiment 1, this increase in speed was apparently achieved in part by trading accuracy for speed, though the higher error rate under auditory-task emphasis may also have been due to the more difficult pitch discrimination required in this condition.
The effect of SOA on digit accuracy ranged from a very substantial $18.8 \%$ under auditory-task emphasis with the short display duration to only $4.9 \%$ with the long display duration in the no-emphasis condition. It is interesting to note that the $18.8 \%$ SOA effect approaches the $19.9 \%$ effect found by Pashler (1989) in an experiment in which the first task also required complex visual processing. The difference in error rate between short and long SOAs was significantly larger when the auditory task was emphasized, especially for the short display duration. These results are consistent with the notion that, without special precautions, the use of a relatively easy first task and a more challenging second task may lead subjects to focus advance preparation on the latter task. Consistent with the present results, such a strategy would hurt first-task performance, but also, and particularly at short SOAs, enable perceptual processing in the second task to proceed while the first task is still in progress.

In this experiment, there was a highly significant positive microdependency between the auditory and digit tasks, especially when the two tasks overlapped extensively (short SOA). Why this dependency also was stronger for the long display duration is unclear. The fact that an overall positive microdependency was obtained suggests that trial-to-trial fluctuations in relative preparatory set, which by themselves would give rise to negative dependencies, were overwhelmed by other factors that, as discussed earlier, would give rise to positive dependencies.

When no task was specifically emphasized, an overall $8.6 \%$ effect of SOA on digit accuracy was found. This effect approaches the 9.5\% SOA effect found in Experiment 1 under digit-task emphasis with well-trained subjects, and it still considerably exceeds the $2.5 \%$ effect found by Pashler (1989). Some factor other than level of practice must therefore explain our failure to replicate these minimal SOA effects.

\section{EXPERIMENT 3}

One potentially important difference between the previous experiments and those by Pashler (1989) concerns the type of mask used to delimit identification of the highest digit. The previous experiments used variable and complex masks constructed by superimposing eight digits

Table 4

Mean Tone Reaction Time as a Function of Task Emphasis, Short Versus Long Display Duration, SOA, and Accuracy of the Digit Response in Experiment 2

Task Emphasis

\begin{tabular}{|c|c|c|c|c|}
\hline \multirow[b]{3}{*}{ Digit Response } & \multicolumn{4}{|c|}{ 200 - } \\
\hline & \multicolumn{2}{|c|}{ Auditory } & \multicolumn{2}{|c|}{ Auditory and Visual } \\
\hline & 50-msec SOA & 1,000 -msec SOA & 50-msec SOA & 1,000 -msec SOA \\
\hline \multicolumn{5}{|c|}{ Short Display Duration } \\
\hline Correct & 401 & 416 & 510 & 509 \\
\hline Incorrect & 426 & 408 & 534 & 514 \\
\hline \multicolumn{5}{|c|}{ Long Display Duration } \\
\hline Correct & 400 & 412 & 493 & 503 \\
\hline Incorrect & 438 & 432 & 536 & 525 \\
\hline
\end{tabular}

Note-RTs are in milliseconds. 
Table 5

Mean Reaction Time (RT), Display Duration, and Error Rates in the Auditory and Visual Tasks as a Function of Mask Type and SOA in Experiment 3

\begin{tabular}{|c|c|c|c|c|}
\hline & \multicolumn{4}{|c|}{ Mask Type } \\
\hline & \multicolumn{2}{|c|}{ Simple } & \multicolumn{2}{|c|}{ Complex } \\
\hline & 50-msec SOA & $1,000-\mathrm{msec}$ SOA & 50-msec SOA & 1,000-msec SOA \\
\hline \multicolumn{5}{|c|}{ Auditory Task } \\
\hline RT & 462 & 447 & 471 & 450 \\
\hline Percent errors & 4.9 & 2.1 & 4.9 & 2.6 \\
\hline \multicolumn{5}{|c|}{ Visual Task } \\
\hline Display duration & 193 & 192 & 233 & 233 \\
\hline Percent errors & 31.2 & 21.6 & 31.9 & 23.0 \\
\hline
\end{tabular}

Note-RTs and display durations are in milliseconds.

Table 6

Mean Tone Reaction Time as a Function of Mask Type, SOA, and Accuracy of the Digit Response in Experiment 3

\begin{tabular}{cccccc}
\hline & \multicolumn{4}{c}{ Mask Type } \\
\cline { 2 - 3 } \cline { 5 - 6 } Digit Response & 50 -msec SOA & 1,000 -msec SOA & & \multicolumn{2}{c}{ Complex } \\
\cline { 2 - 6 } Correct & 460 & 446 & & 467 & 450 \\
Incorrect & 465 & 452 & & 478 & 448 \\
\hline
\end{tabular}

Note-RTs are in milliseconds.

that were randomly selected from the range of 1-5, whereas Pashler (1989) used a constant mask consisting of the letter X. It is known that constant and simple visual patterns may lose their effectiveness as backward pattern masks upon repeated exposure (Breitmeyer, 1984). If the more complex masks used in the previous experiments were more effective in delimiting the period during which visual information was available for perceptual processing, that may explain the larger SOA effect on digit accuracy. In Experiment 3, we tested this possibility by comparing the SOA effects for simple, constant masks with those for complex, variable masks.

\section{Method}

Subjects. Fourteen Ohio State University undergraduates participated for course credit.

Apparatus and Stimuli. These were identical to those of Experiment 2, except for the masks. Complex masks were identical to those used in Experiment 2. Simple masks consisted of the letter $\mathrm{X}$. The two tones in the auditory task were 300 and $900 \mathrm{~Hz}$.

Design. The subjects served in a single session of about $1 \mathrm{~h}$, with 14 blocks of 32 trials each. The first 2 blocks were considered training and were excluded from analysis. Simple masks were used in 1 practice block and complex masks in the other. Mask type was held constant for 3 consecutive experimental blocks, after which it was changed. Half of the subjects started with the simple mask and the other half with the complex mask.

Procedure. The procedure was essentially the same as that for the no-emphasis condition in Experiment 2. Display durations were controlled separately for simple and complex masks, aiming at an overall accuracy of $70 \%$ in the digit task. The display duration was increased by $14 \mathrm{msec}$ after an error in the digit task and decreased by $6 \mathrm{msec}$ after an error. The initial display durations for the two mask types were set at $250 \mathrm{msec}$.

\section{Results}

Mean RTs and percent errors in the auditory task and mean display durations and errors in the digit task are pre- sented in Table 5, for each combination of mask type and SOA.

Auditory (first) task. There were no significant effects of SOA or mask type on RT. Errors were more frequent at the short SOA $[F(1,13)=5.98, p<.03]$.

Digit (second) task. Display durations were significantly longer with the complex masks $[F(1,13)=9.23, p<$ $.01]$. Errors occurred more frequently at the short SOA $[F(1,13)=25.87, p<.001]$. No other effects approached significance.

Table 6 presents mean tone RTs, conditionalized on digit accuracy. There were no significant effects.

\section{Discussion}

As expected, complex masks were more effective than simple masks; the same accuracy in the digit task required significantly longer display durations when complex masks were used. More importantly, however, mask type did not influence the effect of temporal overlap on digit accuracy. The overall effect of SOA was $9.3 \%$, very similar to that obtained in comparable conditions in Experiments 1 and 2. Again, this effect considerably exceeded that reported by Pashler (1989), despite the fact that performance in the auditory task appears to have been very similar. These results make it clear that differences in mask type were not responsible for the discrepancy between the present results and those reported by Pashler (1989).

\section{EXPERIMENT 4}

One remaining plausible explanation for our failure to replicate the minimal effects of temporal overlap on digit accuracy reported by Pashler (1989) lies in the different set of SOAs used. For instance, 50- and 1,000-msec SOAs were used in Experiments 2 and 3, whereas Pashler used 
50-, 150-, and 650-msec SOAs. If the 650-msec SOA frequently provided insufficient time to complete the auditory task and switch preparation to the digit task before the digit display was masked, this would explain the smaller effects of SOA on digit accuracy found by Pashler (1989). To test this possibility, we followed Pashler's (1989) procedure very closely in Experiment 4, using the same masks and the same set of SOAs.

\section{Method}

Subjects. Eighteen Ohio State University undergraduates participated for course credit.

Apparatus and Stimuli. These were the same as in Experiment 3, except that the masks always consisted of the letter $\mathrm{X}$.

Design. The subjects performed 16 blocks of 30 trials each. The first 2 blocks were used for training and were excluded from analysis.

Procedure. The procedure was the same as that of Experiment 3, except for the following changes. Three equiprobable SOAs $(50$, 150 , or $650 \mathrm{msec}$ ) were randomly distributed across the trials in a block. Display duration was controlled by a tracking procedure designed to achieve an overall accuracy of $70 \%$ in the digit task, using the same increments and decrements as used in Experiment 3. Initial display duration was set at $250 \mathrm{msec}$.

\section{Results}

Mean RTs and percent errors in the auditory task and average display durations and errors in the digit task are presented in Table 7 for each of the SOAs.

Auditory (first) task. Tone responses were somewhat slower at the long SOA $[F(2,34)=6.16, p<.01]$, whereas errors were more frequent at short SOAs $[F(2,34)$ $=17.50, p<.001]$.

Digit (second) task. Display durations were the same for all SOAs. The error rate in the digit task decreased by only $5.1 \%$ from the shortest to the longest SOA, though this effect was highly significant $[F(2,34)=15.51, p<.001]$.

In order to evaluate more directly the effects of SOA range on digit accuracy, we compared the results for the simple mask in Experiment 3 with the present results for the 50- and 650-msec SOAs. Thus, the factors in this analysis of variance were SOA range (50-1,000 vs. 50-650 msec, a between-subject factor) and SOA duration (50 vs. 650 or $1,000 \mathrm{msec}$, a within-subject factor). This analysis revealed a highly significant effect of SOA duration $[F(1,30)=53.49, p<.001]$, as well as an interaction of SOA range and SOA duration $[F(1,30)=$

Table 7

Mean Reaction Time (RT), Display Duration, and Error Rates in the Auditory and Visual Tasks as a Function of SOA in Experiment 4

\begin{tabular}{lccc}
\hline & \multicolumn{3}{c}{ SOA } \\
\cline { 2 - 3 } & 50 & 150 & 650 \\
\hline & Auditory Task & & \\
RT & 453 & 451 & 471 \\
Percent errors & 8.1 & 5.2 & 3.8 \\
& Visual Task & & \\
Display duration & 200 & 201 & 202 \\
Percent errors & 30.0 & 29.1 & 24.9 \\
\hline
\end{tabular}

Note-RTs, SOAs, and display durations are in milliseconds.
Table 8

Tone Reaction Time as a Function of Accuracy of the Digit Response in Experiment 4

\begin{tabular}{cccc}
\hline & \multicolumn{3}{c}{ SOA } \\
\cline { 2 - 4 } Digit Response & 50 & 150 & 650 \\
\hline Correct & 450 & 444 & 468 \\
Incorrect & 461 & 466 & 480 \\
\hline
\end{tabular}

Note-RTs and SOAs are in milliseconds.

$5.44, p<.03$ ]. The latter interaction indicates that the difference in digit accuracy between SOAs was significantly larger for the 50 to $1,000-\mathrm{msec}$ SOA range.

Table 8 presents mean tone RTs, conditionalized on digit accuracy. Tone responses were significantly slower when the digit response was incorrect $[F(1,17)=14.58$, $p<.001]$.

\section{Discussion}

The present results replicate quite closely those reported by Pashler (1989) for a very similar experiment. Both experiments showed somewhat longer tone RTs at the longest SOA, a significant effect of SOA on the error rate in the digit task, and a positive dependency between the two tasks. The effect of SOA on the error rate in the digit task was still approximately twice as large in the present experiment $-5.1 \%$ versus $2.5 \%$-however, the rather large standard error for the difference in error rates between SOAs (approximately $1.5 \%$ ) makes this remaining difference seem minimal.

Prolonging the SOA from 650 to $1,000 \mathrm{msec}$ significantly increased digit accuracy. This supports our suggestion that a 650 -msec SOA may frequently provide insufficient time to complete the auditory task and switch preparation to the digit task before the digit display is masked, and that this may explain the previously reported minimal effects of temporal overlap.

It seems reasonable to conclude that the substantially larger SOA effects obtained in the previous experiments with SOAs of 50 and $1,000 \mathrm{msec}$ provide a more accurate indication of the degree to which performance of the digit task is impeded by temporal overlap with an auditory task.

\section{GENERAL DISCUSSION}

Conflicting results have been reported as to whether identification of visually presented characters is impeded by temporal overlap with an auditory task. In order to explain this discrepancy, we distinguished between two sources of interference between overlapping tasks, preparatory limitations and structural limitations of specific processing mechanisms, with the relative importance of these limiting factors depending on the preparatory strategies that subjects adopt. We proposed that the degree to which second-task stimuli can be identified in parallel with another task depends critically on advance preparation for the requisite operations.

The present results provide strong support for this proposal. Inducing subjects to perform the speeded audi- 
tory task at a high level of proficiency, thus forcing them to focus advance preparation on that task, resulted in large detrimental effects of temporal overlap on their ability to identify the highest digit in a briefly presented array. In contrast, when the subjects were encouraged or allowed to focus advance preparation on the digit task, performance of the auditory task deteriorated markedly, whereas the effect of temporal overlap on digit-task accuracy was significantly reduced. However, even in the latter case, we observed substantial effects of temporal overlap when the longest SOA provided sufficient time to complete the auditory task and to switch preparation to the digit task before the digit display was presented. These results are consistent with Pashler's (1989) conclusion that perceptual processing can operate in parallel with the performance of another task. They also show, however, that perceptual identification is impeded even when the overlapping task imposes only minimal perceptual requirements, and they identify preparatory limitations as the major cause of such interference.

Results similar to those reported here have been presented by McLeod and Posner (1984), who combined an auditory-verbal task (e.g., saying "high" when hearing the word high) with a visual same-different task. They found that the highly compatible speech task showed no slowing at the times when the visual stimuli were arriving, compared with the times when these stimuli were merely expected to arrive. Yet, the speech task was much faster when performed alone, that is, when the subjects knew that no visual stimuli would arrive. In accordance with our data, these results indicate that the interference between the two tasks was of preparatory origin and did not result from structural limitations in parallel-task performance.

\section{The Interpretation of \\ Overlapping-Task Interference}

The present results indicate that preparatory limitations can be important in overlapping-task performance. In fact, these results are consistent with the notion, first proposed by Gottsdanker (1979), that preparatory factors may in fact be solely responsible for interference between overlapping tasks when advance preparation is restricted to the first task. This view does not deny or disregard the existence of structural, resource-like, or bottleneck-like limitations in perception and decision; there is ample evidence that such limitations exist. However, we propose that structural limitations should be invoked in overlappingtask performance only when both tasks are prepared in advance or when preparatory limitations are otherwise circumvented (Duncan, 1980; Pashler, in press), because it is only under such circumstances that processing mechanisms would be called upon to service two tasks at a time.

Though it is important to distinguish between preparatory and structural limiting factors in overlapping-task performance, we believe that these factors may be closely related. De Jong (in press) presented evidence that subjects prepare for each task in turn, in a specific order, when both tasks require a speeded response. Such a strategy is likely to be optimal in the sense that it minimizes the total time required to complete both tasks. The alternative strategy of dividing advance preparation between the two tasks would merely result in slower performance of the first task, and thus in prolonged postponement of selection of the second response until the responseselection bottleneck is cleared by the first task. Thus, the sequential preparatory strategy should, in this case, be viewed as an appropriate way of coping with the severe structural limitations of the response-selection mechanism. When, as in the experiments reported here, the second task requires the identification of briefly presented stimuli but no speeded response, preparing both tasks in advance will allow subjects to trade speed in the first task for greater accuracy in the second task. In many cases, this may be an appropriate compromise, and it is probably no coincidence that strong manipulations were needed in order to induce subjects to adopt a more sequential preparatory strategy.

These considerations suggest that preparatory limitations and preparatory strategies should be distinguished in overlapping-task performance. Preparatory limitations refer to the fact that people have a limited capacity for preparing multiple tasks in advance (De Jong, in press; Gottsdanker, 1980; Logan, 1978). Such limitations constitute a general source of interference between overlapping tasks. Preparatory strategies serve to allocate preparation to overlapping tasks in such a manner as to avoid, when possible, simultaneous demands on bottleneck-like processing mechanisms and to exploit, when appropriate, possibilities for parallel processing provided by resourcelike mechanisms. This theoretical approach stresses the interrelations between preparatory, strategic, and structural limiting factors in overlapping-task performance, and can at least qualitatively account for many recent findings, including the present ones, that are difficult to explain in terms of only preparatory or structural limitations (De Jong, 1993, in press; Gottsdanker, 1979, 1980; Pashler, 1989, in press). It also suggests that a more complete understanding of the sources of limitations in overlappingtask performance may require examining performance at several different levels of relative task emphasis, perhaps along the lines suggested by Navon (1985) for other dualtask paradigms.

Finally, we found positive microdependencies between the two overlapping tasks in at least two of the experiments, in marked contrast to the negative macrotradeoffs observed in the first two experiments. We have already indicated the most likely explanation for this finding. Microdependencies are determined not only by trial-totrial fluctuations in relative task emphasis, but also by fluctuations in the overall level of preparedness and by temporal dependencies between the processing operations in the two tasks. The latter two factors appear to predominate, causing overall microdependencies to be positive. Microdependencies thus seem unsuited for evaluating possible effects of preparatory strategies on overlapping-task performance; such effects are best assessed by systematic manipulation of preparatory strategies through explicit instructions. 


\section{REFERENCES}

BREITMEYER, B. G. (1984). Visual masking: An integrative approach. London: Oxford University Press.

DE JoNG, R. (1993). Multiple bottlenecks in overlapping task performance. Journal of Experimental Psychology: Human Perception \& Performance, 19, 965-980.

DE JONG, R. (in press). The role of preparation in overlapping task performance. Quarterly Journal of Experimental Psychology.

Duncan, J. (1980). The locus of interference in the perception of simultaneous stimuli. Psychological Review, 98, 433-458.

EriKsen, C. W. (1980). The use of a visual mask may seriously confound your experiment. Perception \& Psychophysics, 28, 89-92.

GoTrSDANKER, R. (1979). A psychological refractory period or an unprepared period? Journal of Experimental Psychology: Human Perception \& Performance, 5, 208-215.

GoTTSDANKER, R. (1980). The ubiquitous role of preparation. In G. E. Stelmach and J. Requin (Eds.), Tutorials in motor behavior (pp. 315371). Amsterdam: North-Holland.

Kahneman, D. (1973). Attention and effort. Englewood Cliffs, NJ: Prentice-Hall.

LOGAN, G. D. (1978). Attention in character classification: Evidence for the automaticity of component stages. Journal of Experimental Psychology: General, 107, 32-63.

MCCanN, R., \& Johnston, J. C. (1992). Locus of the single-channel bottleneck in dual-task interference. Joumal of Experimental Psychology: Human Perception \& Performance, 18, 471-485.

McLeod, P. (1977). Parallel processing and the psychological refractory period. Acta Psychologica, 41, 381-396.

MCLeod, P., \& PosNer, M. I. (1984). Privileged loops from percept to act. In H. Bouma \& E. G. Bouwhuis (Eds.), Attention and performance $X$ (pp. 55-66). Hillsdale, NJ: Erlbaum.
Navon, D. (1985). Attention division or attention sharing? In M. I. Posner \& O. S. M. Marin (Eds.), Attention and performance XI (pp. 251-266). Hillsdale, NJ: Erlbaum.

PASHLER, H. (1984). Processing stages in overlapping tasks: Evidence for a central bottleneck. Joumal of Experimental Psychology: Human Perception \& Performance, 10, 358-377.

PAShler, H. (1989). Dissociations and contingencies between speed and accuracy: Evidence for a two-component theory of divided attention in simple tasks. Cognitive Psychology, 21, 469-514.

Pashler, H. (in press). Overlapping mental operations in serial performance with preview. Quarterly Journal of Experimental Psychology.

Pashler, H., \& Johnston, J. C. (1989). Chronometric evidence for central postponement in temporally overlapping tasks. Quarterly Journal of Experimental Psychology, 41A, 19-45.

SCHWEICKERT, R. (1978). A critical path generalization of the additive factors method: Analysis of a Stroop task. Joumal of Mathematical Psychology, 18, 105-139.

WELFORD, A. T. (1980). The single-channel hypothesis. In A. T. Welford (Ed.), Reaction time (pp. 215-252). New York: Academic Press.

\section{NOTE}

1. Due to the trial-by-trial tracking procedure, display durations for correct digit-task responses exceeded those for incorrect responses by about 20 msec on the average. This factor would also tend to blur existing microdependencies between the two tasks.

(Manuscript received November 30, 1992; revision accepted for publication May 21, 1993.) 